\title{
Correlating familial Alzheimer's disease gene mutations with clinical phenotype
}

\author{
Natalie S Ryan and Martin N Rossor \\ University College London, Institute of Neurology, London, UK (N.S. Ryan, M.N Rossor) \\ Correspondence: N S Ryan, Dementia Research Centre, Department of Neurodegenerative \\ Diseases, University College London, Institute of Neurology, Queen Square, London WC1N 3BG, \\ UK. Tel: +44 (0) 08451555000 Fax: +44 (0) 2076762066 ryan@drc.ion.ucl.ac.uk
}

\begin{abstract}
Alzheimer's disease (AD) causes devastating cognitive impairment and an intense research effort is currently devoted to developing improved treatments for it. A minority of cases occur at a particularly young age and are caused by autosomal dominantly inherited genetic mutations. Although rare, familial AD provides unique opportunities to gain insights into the cascade of pathological events and how they relate to clinical manifestations. The phenotype of familial AD is highly variable and, although it shares many clinical features with sporadic AD, it also possesses important differences. Exploring the genetic and pathological basis of this phenotypic heterogeneity can illuminate aspects of the underlying disease mechanism, and is likely to inform our understanding and treatment of $\mathrm{AD}$ in the future.
\end{abstract}

Keywords: Familial Alzheimer's disease, Presenilins, Amyloid precursor protein, early onset dementia, Dominantly inherited Alzheimer's disease 


\section{Introduction}

Observations that cases of early onset Alzheimer's disease (EOAD) may cluster within families were made as early as the 1930s, and almost two decades have now passed since the first reports confirming that the disease could be caused by autosomal dominantly inherited genetic mutations. Initially identified in the amyloid precursor protein (APP) gene on chromosome $21,{ }^{(1)}$ mutations were also subsequently found in the genes encoding presenilin one (PSEN1) on chromosome $14^{(2)}$ and presenilin two (PSEN2) on chromosome 1. ${ }^{(3)}$ Over two hundred changes have now been described in these genes, although some are thought to represent non-pathogenic polymorphisms and in other cases, the pathogenic nature of the change is as yet unclear. More recently, duplications of the APP gene have been identified as an additional cause of familial Alzheimer's disease (FAD). ${ }^{(4)}$ Mutations in PSEN1 account for the majority of cases of FAD and it is in PSEN1 that the greatest numbers of mutations have been found, with 177 recorded to date compared to 32 and 14 in APP and PSEN2 respectively. ${ }^{(102)}$ (See Figures 1,2 and 3) Collectively, these mutations are thought to account for up to $0.5 \%$ of Alzheimer's disease in general. ${ }^{(102)}$ The degree to which familial Alzheimer's disease may be a paradigm for the commoner, late onset, sporadic form of the disease is uncertain but the insights gleaned from the study of FAD have catalysed much of our current knowledge of the pathobiological basis of the disease. The development of cellular models and transgenic mice harbouring mutations in APP and the presenilins has allowed the disease mechanism to be explored in detail and probed with potential therapeutic agents. As these therapies come closer to clinical application, the debate regarding how generalisable the changes of FAD are to those of sporadic AD grows in significance. Unravelling the pathway through which specific genetic mutations translate into the manifestations of clinical Alzheimer's disease, and how the resulting phenotypes may vary, are clearly important areas of investigation. Although much has been published describing novel FAD gene mutations and exploring their functional effects at a molecular level, there have been far fewer detailed reports of the resulting clinical phenotypes. ${ }^{(5)}{ }^{(6)}$ In this review, we examine how closely the phenotype of FAD resembles and differs from that of sporadic AD. We emphasise the importance of appreciating the degree of phenotypic heterogeneity within FAD. Finally, we 
discuss several mechanisms by which the clinical picture, and underlying pathology, may be influenced by genotype.

\section{The role of genetic mutations in $\mathrm{AD}$ pathogenesis}

The pathological hallmarks of Alzheimer's disease in both familial and sporadic cases are extracellular plaques of amyloid- $\beta(\mathrm{A} \beta)$ and intraneuronal inclusions of hyperphosphorylated tau. It is now well recognised that mutations in all three genes known to cause FAD ultimately result in enhanced production and/or deposition of A $\beta .{ }^{(7)}$ The mechanisms through which they achieve this are complex and variable. APP is a transmembrane protein whose function is not yet fully determined, although it appears to play a role in neural plasticity and the regulation of synapse formation. APP may be proteolytically cleaved by $\alpha, \beta$ and $\gamma$ secretases and, during its normal metabolism, it can undergo cleavage via alternative pathways. Cleavage by $\alpha-$ secretase is considered to be part of the non-amyloidogenic pathway in APP processing. It precludes $A \beta$ formation as it cleaves within the segment of APP that would otherwise give rise to A $\beta$. Upon cleavage by $\alpha$-secretase, APP releases its extracellular domain, a fragment called APPs $\alpha$, which is known to have neurotrophic effects. Alternatively, APP may undergo sequential cleavage by $\beta$ and $\gamma$ secretase. Extracellular cleavage by $\beta$-secretase (also known as BACE1: $\beta$-site of APP cleaving enzyme) generates a soluble extracellular fragment and is followed by cleavage of APP within its transmembrane domain by $\gamma$ secretase. The unusual property of $\gamma$ secretase is that it may cleave APP at a variety of different sites, generating a peptide from 39-43 amino acids in length; the amyloid- $\beta$ (A $\beta$ ) protein. PSEN1 forms part of the $\gamma$ secretase complex and the majority of pathogenic PSEN1 mutations are located in areas thought to lie in the vicinity of its transmembrane domains. Under normal conditions, the majority of $A \beta$ takes the form of the 40 amino acid long peptide $A \beta 40$. There is some additional production of the longer moiety; $A \beta 42$, which accounts for $5-15 \%$ of the total pool of $A \beta$ in health. Smaller quantities of other $A \beta s$, both longer and shorter, may also be observed. A $\beta$ peptides, particularly $A \beta-42$, can cause toxic effects on neuronal and synaptic function as intracellular oligomers and seed to form extracellular precipitates in the form of amyloid plaques. $A \beta-42$ is the most amyloidogenic form of the peptide; its hydrophobic nature makes it more susceptible 
to conformational change leading to amyloid fibrillogenesis. Most FAD-causing mutations appear to either increase $A \beta-40$ and $A \beta-42$ or alter the ratio between them. However, the discovery of the 'Arctic' APP mutation (E693G) in a Swedish family, which is located away from the secretase sites, led to the suggestion of an alternative pathogenic mechanism. $A \beta-40$ and $A \beta-42$ are actually found in decreased levels with this mutation, however they have an increased propensity to form protofibrils, which may accelerate their deposition in intracellular or extracellular locations, or both. ${ }^{(8)}$

\section{Figure 1. APP gene with mutations indicated}

Diagram courtesy of Richard Crook, Mayo Clinic Jacksonville, and John Hardy, Institute of Neurology, University College London.

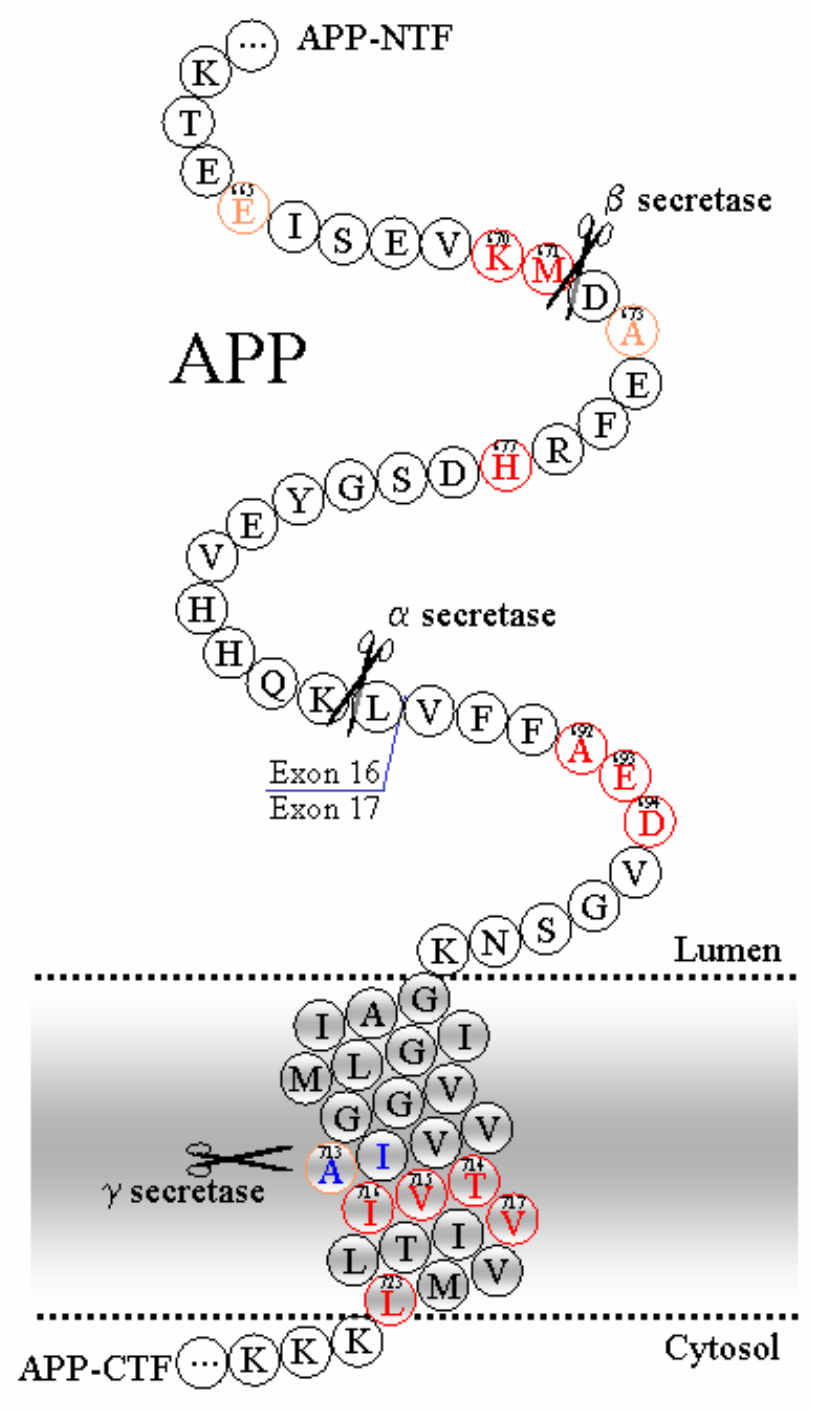




\section{Figure 2. Presenilin 1 gene with mutations indicated}

Reprinted from International Review of Cytology, Vol 254, Dillen K. \& Annaert W., A two decade contribution of molecular cell biology to the centennial of Alzheimer's disease: are we progressing towards therapy?, Pages 215-300, Copyright (2006), with permission from Elsevier.

FAD-linked mutations are indicated in red, while non-pathogenic mutations are marked in orange. Interaction domains with APP/TLN or NCT/APH-1/PEN-2 are marked in blue, as well as the conserved residues D257 and D385 forming the putative catalytic site. Interactions of the $\mathrm{C}$-terminal domain and the hydrophilic loop domain with proteins such as the brain G-protein Go, calsenilin, the PSEN1associated protein (PSAP), b- and d-catenin, p0071 and PLD1, are shown in dark green. The endoproteolytic cleavage site separating PSEN1-NTF and-CTF in the seventh hydrophobic region, the SPP cleavage site in the ninth TMD and the caspase cleavage site in the hydrophilic loop domain are indicated by yellow arrows.

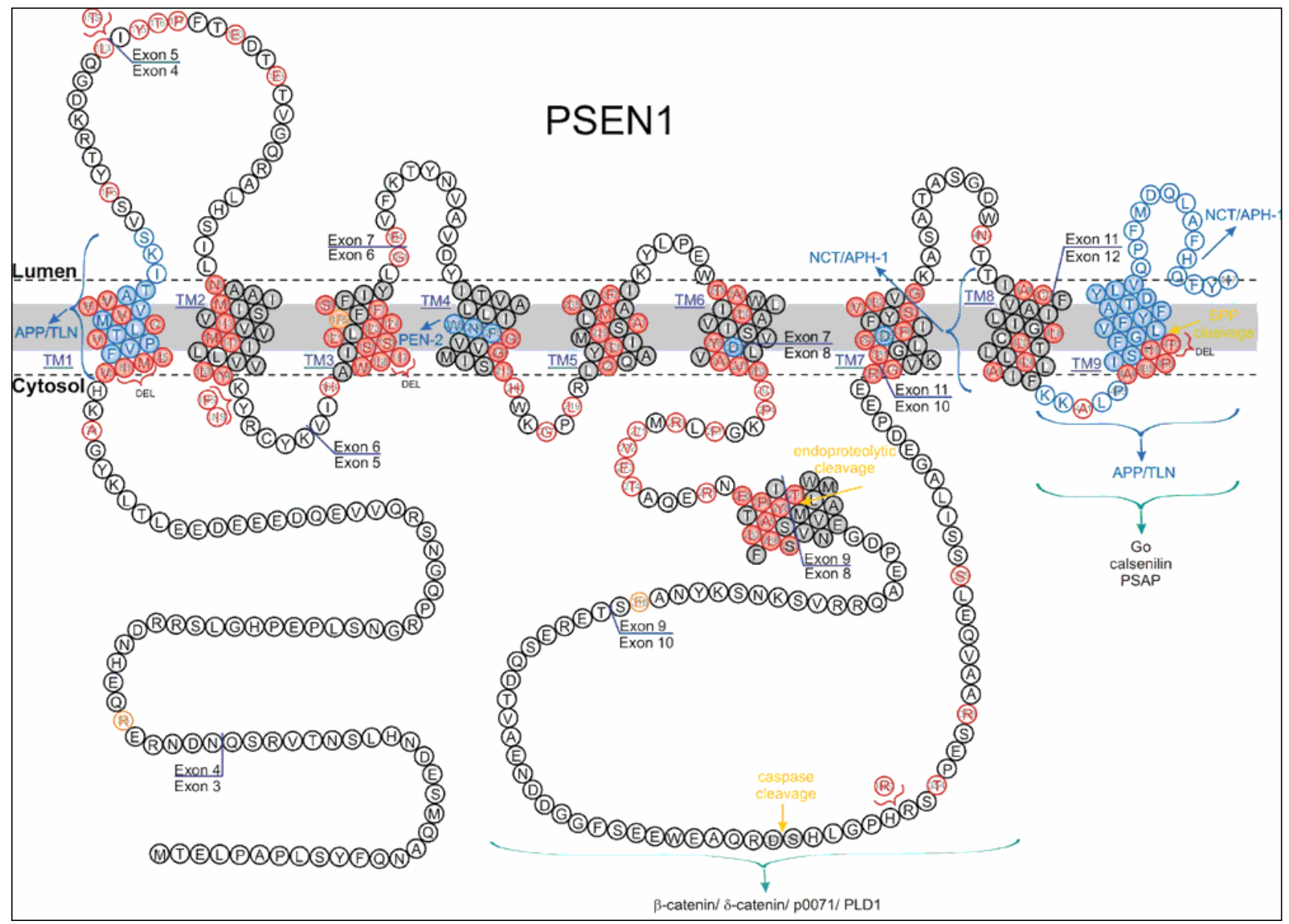




\section{Figure 3. Presenilin 2 gene with mutations indicated}

Diagram courtesy of Richard Crook, Mayo Clinic Jacksonville, and John Hardy, Institute of Neurology, University College London.

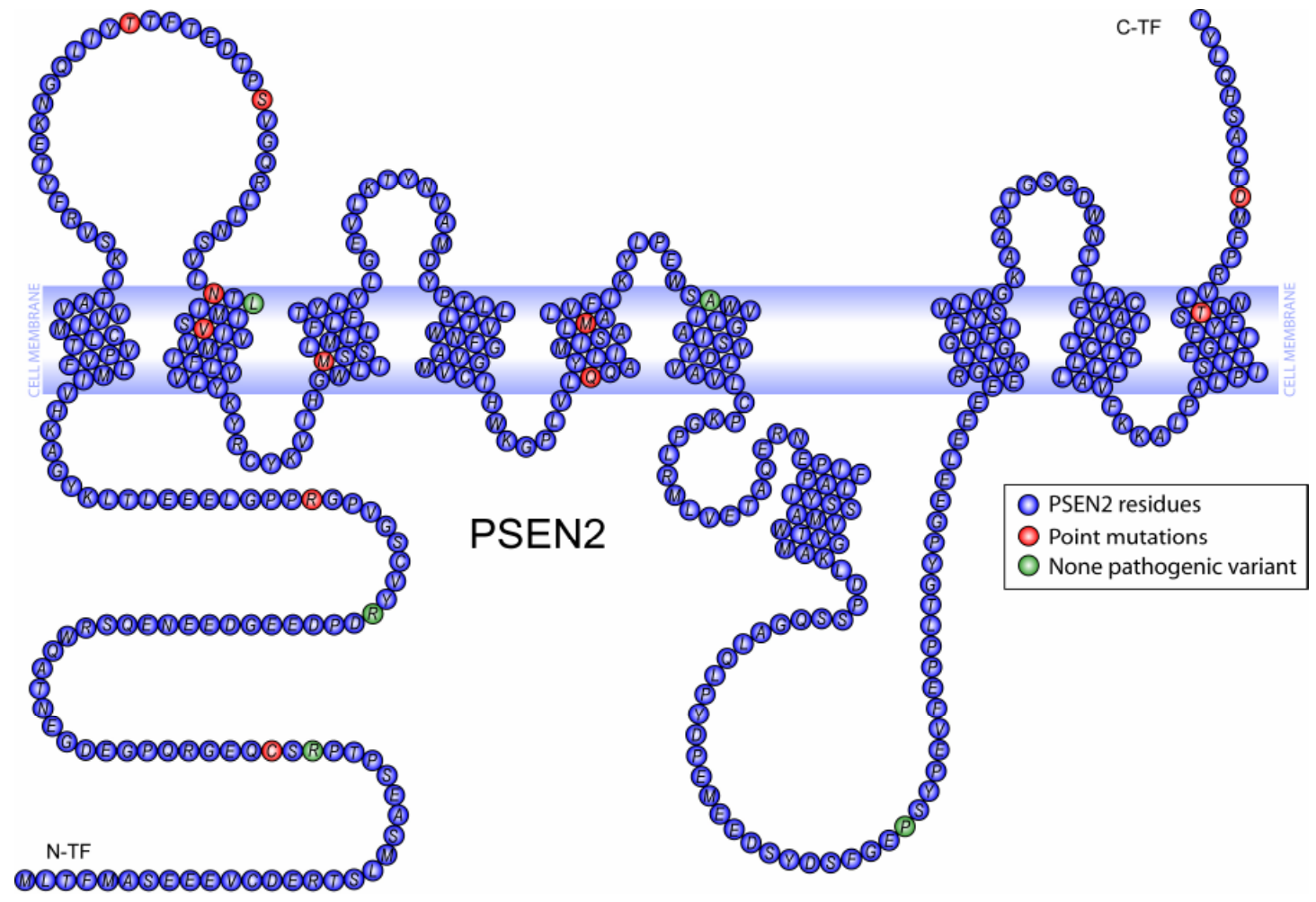

\section{Clinical manifestation of disease}

The most striking difference between familial and sporadic AD is the younger age at onset in individuals harbouring FAD mutations. That the disease should manifest at an earlier age is not surprising, given the increased $\beta$-amyloid deposition that these mutations cause. The youngest ages at onset are seen in families with PSEN1 mutations, which typically fall within the range 35-55 years. APP mutations tend to give rise to symptom onset between 40-65 years and PSEN2 mutations between 40-70 years. It has been suggested that, as well as being associated with a younger age at 
onset of disease, PSEN1 mutations give rise to shorter disease durations (4.8-6.8 years) than PSEN2 and APP mutations (range $4.4-10.8$ years and $9.0-16$ years respectively). ${ }^{(9)}$ However, the survival data of different series demonstrate considerable variation. Although the disease course of FAD is often considered to be more aggressive, some reports find comparable disease durations for the familial and sporadic forms of AD. ${ }^{(10)}$ Drawing direct comparisons between disease duration in familial and sporadic AD is problematic as survival is likely to be affected by age at onset itself. Younger, healthier patients may survive longer at more severe stages of disease than older patients with sporadic AD, who are generally more frail and often have additional co-morbidities. Another major difficulty in interpreting survival data is that estimates for age at symptom onset are highly subjective and unreliable. Symptoms tend to develop insidiously so, in some cases, may pass unrecognised for quite some time. On the other hand, as awareness of the heritable nature of the disease heightens with subsequent generations, symptoms are likely to be noticed at an earlier stage. This probably contributes to the range in age at onset that is witnessed both between and within families with the same mutation. However, other genetic and epigenetic factors are also likely to be important in modulating age at onset.

\section{Other genetic modifiers}

Pathological studies have shown that the proportion of brain tissue occupied by A $\beta$ 42-containing plaques is significantly higher in PS1-associated AD than sporadic AD. Although there does not appear to be a difference in the amount of tissue containing A $\beta-40$ between these two disease groups, both PS1-AD and sporadic AD show the same relationship between $A \beta$ deposition and APOE genotype: Individuals who carry an E4 allele demonstrate a greater proportion of $A \beta-40$ deposition in both familial and sporadic disease. It has been suggested that, in the presence of the apolipoprotein E4 isoform, the threshold for $A \beta-40$ fibrillisation is decreased, fostering the deposition of $\mathrm{A} \beta-40$ onto $A \beta-42$-containing plaques. ${ }^{(11)}$ It has also been shown that possession of the APOE4 allele in FAD secondary to APP mutations is associated with a decreased age at onset, as is the case in sporadic AD. In PSEN1 mutations, this modifying effect on age at onset by the E4 allele was not previously thought to occur. ${ }^{(12)}$ However, when a single large Colombian kindred with the E280A PSEN1 mutation was examined, possession of the APOE4 allele did appear to be associated with an earlier 
age at onset. ${ }^{(13)}$ The true nature of the relationship between APOE status and FAD mutations will probably only be elucidated when much larger cohorts are studied.

\section{Cognitive phenotypes}

\section{Amnestic presentations}

The majority of individuals with FAD have similar clinical presentations to those with sporadic AD, comprising early impairment of episodic memory which gradually progresses to involve multiple cognitive domains. Longitudinal studies of individuals at risk of FAD demonstrate that the earliest neuropsychological manifestations are a fall in verbal memory and performance IQ scores, occurring even 2-3yrs presymptomatically. ${ }^{(14)}$ The neuropsychological profile of FAD at a group level has also been found to show relatively well preserved naming, spelling and visuoperceptual skills compared with sporadic AD in the early stages. ${ }^{(15)}{ }^{(10)}$ There can therefore be a particularly long prodromal phase in FAD, where subtle memory problems are the only clinical feature for several years. ${ }^{(10)}$ Presymptomatic cognitive deficits are accompanied by neuroimaging changes, with accelerating rates of whole brain and hippocampal atrophy in the five years prior to symptom onset. ${ }^{(16)(17)}$

The early stages of sporadic AD have been harder to study. Historically, individuals with memory concerns have tended to seek medical attention only when their symptoms are well established. However, we are currently witnessing a change in the tide of public opinion towards an appreciation of the importance of early diagnosis. There is increased recognition that most patients with sporadic AD are likely to have experienced a period of relatively isolated, usually amnestic, deficits termed mild cognitive impairment (MCI) prior their diagnosis of AD. ${ }^{(18)}$ The term MCI is usually used to refer to individuals with cognitive decline which is more than anticipated from normal aging but insufficient to fulfil criteria for dementia. The term will thus be applied to a heterogeneous group, not all of whom will develop $\mathrm{AD}$, and there is currently a great research effort aimed at better characterising these individuals and refining the definition of the MCI label. Nevertheless, there are now many lines of 
evidence supporting the notion that sporadic AD is preceded by a long prodromal stage. The canonical FAD phenotype, manifesting with early progressive impairment of episodic memory, can perhaps then be viewed as equivalent to the typical amnestic AD phenotype, preceded by MCI, which is seen commonly in sporadic disease.

Many atypical presentations of FAD have, however, been reported and there can be significant heterogeneity both between and within families regarding clinical phenotype. Heterogeneity, however, also exists within sporadic AD and as recognition of these more unusual variants increases, they may be found to occur more frequently than was previously appreciated.

\section{Language presentations}

Several PSEN1 mutations have been reported to present with language impairment including G209V, H163Y and R278I, which was detected in one patient given an initial clinical diagnosis of progressive non-fluent aphasia. ${ }^{(19)}$ Language deficits have been described in association with many other mutations later in the disease course, as is also often the case in sporadic AD. There is a growing body of research aiming to better characterise individuals with neurodegenerative disease who have predominant impairments of language function at presentation. Primary progressive aphasia (PPA) is traditionally thought to signify a disease in the spectrum of frontotemporal lobar degenerations. However, a 'logopenic' variant (LPA) of PPA has recently been proposed, in which $\mathrm{AD}$ appears to be the commonest underlying pathology. ${ }^{(20)}$

\section{Behavioural and psychiatric presentations}

In sporadic $\mathrm{AD}$, symptoms of agitation, depression, delusions and hallucinations occur commonly, particularly in the later stages of disease. Many PSEN1 mutations are associated with behavioural and psychiatric symptoms during the course of the disease and these can, rarely, be the presenting features. Prominent early behavioural symptoms have also been reported in association with several PSEN2 mutations, including M239V, T122R and Y231C. ${ }^{(21)}$ An underlying pathological diagnosis of AD has been obtained for some PSEN1 mutations where a behavioural presentation has been described, including V89L ${ }^{(22)}, \mathrm{L}_{226 \mathrm{~F}^{(23)}}$ and M139V ${ }^{(24)}$. It has been suggested that a distribution of pathology involving structures in frontal-subcortical 
circuits, such as the thalamus and striatum, may contribute to the behavioural phenotype in some of these patients. ${ }^{(22)}$ Pathological confirmation for other PSEN1 mutations such as L113P remains to be established. ${ }^{(25)}$ In some cases, the presence of behavioural and language features has been prominent enough to prompt a clinical diagnosis of frontotemporal dementia (FTD), as was the case for the PSEN1 insR352 genotype. ${ }^{(26)(27)}$ It was initially hypothesised that this PSEN1 genotype may be capable of inducing a distinct neurodegenerative illness, more akin to FTD than AD. Intriguingly, not only did the members of the kindred have an FTD phenotype but when an affected individual eventually came to autopsy, ubiquitin pathology typical of FTD was found. The subsequent discovery of the progranulin gene as a cause of familial FTD led to a re-evaluation of this case, which was indeed found to harbour a progranulin mutation. ${ }^{(28)}$ With the benefit of hindsight, the change in PSEN1 was then reconsidered to be a non-pathogenic polymorphism. This case highlights the importance of exercising caution when ascribing pathogenicity to rare PSEN1 variants, especially when the phenotype is unusual.

\section{Visual presentations}

Visuospatial and visuoperceptual deficits commonly occur in the later stages of both sporadic and familial $\mathrm{AD}$. In sporadic $\mathrm{AD}$, a posterior cortical atrophy variant may occur, where marked visual processing deficits are the presenting features and memory is relatively well preserved until later in the disease. ${ }^{(29)}$ This phenotype has not, to date, been observed in FAD.

\section{Neurological symptoms and signs}

Neurological symptoms and signs tend to be more prominent in FAD than sporadic $\mathrm{AD}$, and have been recognised as a feature of the disease ever since it was first reported. Myoclonus and seizures are common. Spastic paraparesis, extrapyramidal signs and cerebellar ataxia have also been described, though less frequently. All of these features are particularly prominent in kindreds with very early onset of symptoms, below the age of $40 .^{(30)}$ However, it is important to note that significant phenotypic heterogeneity is observed, both between families sharing a common 
mutation and within the affected individuals of a single family. Whilst all of these unusual features do appear to occur more frequently in familial than sporadic AD, the fact that younger patients often survive to more severe stages of disease may contribute in part to the trend. Furthermore, the preference for reporting atypical cases of FAD over those with sporadic AD may also play a role. In some reports, particularly those including longitudinal assessments, it is clear that neurological symptoms and signs developed at an early stage of the disease and there are cases where these features predate cognitive impairment (Table 1). However, other reports of 'early' atypical features should be read with caution as sometimes the disease may be well established by the time a patient first presents to a clinician.

\section{Myloclonus and seizures}

Myloclonus can be a particularly prominent and early sign in FAD, in contrast to sporadic $\mathrm{AD}$ where it tends to become apparent later in the course of the disease. The same pattern appears to occur with seizures, which can occur more frequently and at an earlier stage in the disease in FAD than in sporadic AD. ${ }^{(31)}$ Myoclonus and seizures are observed more often in very early onset cases of FAD. In fact, in one family with the PSEN1 L166P mutation and a young age at onset between 29-35 years, tonic-clonic seizures pre-dated the onset of cognitive impairment. ${ }^{(32)}$ Fine finger myoclonus has also been noted to occur prior the onset of objective memory impairment, although it may not necessarily be apparent to the patient or their family at this mild early stage. ${ }^{(10)}$ Interestingly, there is a cluster of mutations associated with early myoclonus and seizures in the region encoding transmembrane domain III of the PSEN1 gene (S169L, S169P, S170F). Early myoclonus and seizures are also observed in individuals with APP mutations and particularly with APP duplications, see below.

\section{Spastic paraparesis}

Spastic paraparesis has been reported in association with over twenty different PSEN1 mutations. ${ }^{(33)}$ Symptoms of spastic paraparesis may occur simultaneously with cognitive deficits or may predate them by several years. Progressive spastic paraparesis occurring for over a decade prior to the onset of cognitive impairment has been reported in association with the PSEN1 R278K mutation. ${ }^{(34)}$ There is a particular association of spastic paraparesis with PSEN1 exon 9 deletions, although not all 
individuals with these deletions demonstrate the phenotype. ${ }^{(35)}$ In those who do, there are frequently characteristic histological findings of large, rounded plaques in the frontal cortex known as 'cotton wool plaques'. ${ }^{(36)}$ Composed of $A \beta 40$ and 42, cotton wool plaques usually lack a compact amyloid core and show very little neuritic change or glial cell component. The spastic paraparesis phenotype of FAD is often not associated with any specific imaging findings. However, significant white matter abnormalities on cranial MRI, presumed secondary to amyloid angiopathy, have been observed in a family exhibiting spastic paraparesis and cotton wool plaques secondary to the E280G PSEN1 mutation. ${ }^{(37)}$

Several hypotheses have been proposed to explain why certain PSEN1 mutations may give rise to the spastic paraparesis phenotype. The observation that most of the mutations associated with spastic paraparesis cause exceptionally high levels of $A \beta 42$ suggests that a dosage effect may be operating. ${ }^{(38)}$ Intraneuronal A $\beta$ may also play a role; this is a more frequent finding in $\mathrm{AD}$ patients with cotton wool plaques. As intraneuronal $A \beta$ is known to induce only a weak immune response, it has been postulated that cotton wool plaques may result from a combination of increased $A \beta$ production and decreased clearance. ${ }^{(39)}$ However, the presence of cotton wool plaques per se is not enough to explain the phenotype as these have also been found in patients lacking signs of spastic paraparesis. ${ }^{(40)}$ Furthermore, the presence of spastic paraparesis is highly variable, even between the affected individuals of a single family sharing identical mutations and similar environmental exposures. There may, therefore, be additional as yet unidentified factors operating in these patients that drive the pathology towards structures, like the corticospinal tracts and motor cortex, which are usually spared in both familial and sporadic AD.

\section{Extrapyramidal signs}

Extrapyramidal signs have been reported in association with several different PSEN1 mutations, particularly those causing very early onset disease and other neurological signs such as spastic paraparesis. ${ }^{(41)}$ A novel PSEN2 mutation (A85V) has also recently been reported where Parkinsonism was a feature ${ }^{(42)}$ In the majority of cases, the Parkinsonism does not become apparent until several years into the clinical course. However, an extrapyramidal syndrome characterised by rigidity, bradykinesia 
and an abnormal posture and gait was present from the onset of cognitive symptoms in the first reported kindred harbouring the PSEN1 G217D mutation. ${ }^{(43)}$ The autopsy findings in this family included, in addition to neurofibrillary tangles, amyloid angiopathy and neuritic plaques, neuronal rarefaction in the substantia nigra and numerous cotton wool plaques. These were distributed throughout the cerebral cortex but were also concentrated in subcortical structures, particularly the striatum. As the authors suggest, the topography of pathology in these patients could account for their clinical presentations. Furthermore these cases illustrate how the separate, yet often overlapping, phenotypes of spastic paraparesis and Parkinsonism in FAD may share a common pathological substrate of cotton wool plaques located in specific anatomical areas.

Another histological feature that can underlie a Parkinsonian presentation of FAD is Lewy body pathology (LBP). Both LBP and cotton wool plaques were detected in a patient exhibiting a dopa-responsive Parkinsonian syndrome who developed cognitive symptoms seven years after the onset of his movement disorder. He was found to possess a novel three base pair deletion (DelT440) in PSEN1. ${ }^{(44)}$ Widespread neocortical LBP has been demonstrated in a kindred with very early onset FAD secondary to an S170F PSEN1 mutation, in which the age at symptom onset was as young as $26 .{ }^{(30)}$ In this family, myoclonus, seizures and extrapyramidal signs were features of the disease. Parkinsonism, seizures, a very early onset of disease and LBP were also reported in association with the M233V mutation in PSEN1. ${ }^{(45)}$ However, not all FAD patients with extrapyramidal signs are found to have LBP and equally, many patients with LBP do not manifest Parkinsonism clinically. In fact, concomitant LBP is a frequent finding in neuropathological examinations of patients with both familial and sporadic AD, occurring in up to $60 \%$ patients with each disease according to some series. ${ }^{(46)}$ (47) Studies including analysis of the frequency of LBP in different FAD gene mutations have suggested that PSEN1 mutations are significantly more likely to have associated LBP than PSEN2 mutations. ${ }^{(48)}$ Furthermore, there appear to be mutation-specific effects on the development of LBP in FAD. Certain PSEN1 mutations such as A260V are associated with diffuse LBP whereas in others, like G209V, the LBP seems to occur in the amygdala only. In other PSEN1 mutations, such as A246E, there tends to be very little LBP at all. ${ }^{(46)} \mathrm{LBP}$ has also been observed in association with APP mutations and, with both the Presenilins and 
APP, no correlations between mutation position and the development of this pathological picture have been identified. ${ }^{(45)}$ It has been suggested that the presence of diffuse LBP in FAD is associated with longer durations of disease than when the LBP is restricted to limbic areas. ${ }^{(48)}$ However, a detailed understanding of the significance of LBP in FAD and how LBP relates to duration and manifestation of disease remains to be elucidated, particularly as many studies were conducted before synuclein antibodies became available.

\section{Cerebellar signs}

Variable cerebellar $A \beta$ deposition has been observed in both familial and sporadic AD. ${ }^{(49)(50)}$ In several PS1 mutations, particularly E280A, ${ }^{(51)} \mathrm{I} 143 \mathrm{~T},{ }^{(52)} \mathrm{M} 139 \mathrm{~V}^{(53)}$ and $\mathrm{G} 209 \mathrm{~V}^{(54)}$ severe involvement of the cerebellum by $\mathrm{A} \beta$ plaque deposition has been noted. This has also been found in FAD secondary to the APP717 mutations ${ }^{(55)}$ and in the brains of elderly patients with Down's syndrome. ${ }^{(56,57)}$ Even in cases of FAD with similar amounts of $\mathrm{A} \beta$ deposition to sporadic $\mathrm{AD}$ in the cerebellum, cerebellar Purkinje cell loss and reactive astrocytosis are significantly more severe in FAD than sporadic AD. ${ }^{(58)}$ Pathological involvement of the cerebellum may occur without cerebellar signs featuring in the clinical presentation. ${ }^{(5)}$ However, progressive cerebellar ataxia has been reported as preceding cognitive impairment in the S170F ${ }^{(59)}$ and P117A ${ }^{(60)}$ mutations in PSEN1. Furthermore, cerebellar signs have been observed during the disease course in a range of other mutations in PSEN1, including L282V. ${ }^{(61)}$ This mutation is notable for its association with an unusually high amount of amyloid angiopathy, which was found to involve the cerebellum in the individual manifesting cerebellar signs. 


\section{Table 1. Neurological presentations of familial Alzheimer's disease}

Neurological symptoms and signs occur commonly in FAD and may, more rarely, represent the initial clinical features, as summarised below.

\begin{tabular}{|c|c|c|}
\hline Clinical Feature & Mutation & Unusual pathology \\
\hline $\begin{array}{l}\text { Seizures and } \\
\text { myoclonus }\end{array}$ & $\begin{array}{l}\text { PSEN1 N135S } \\
\text { PSEN1 L166P } \\
\text { PSEN1 L235P } \\
\text { 'London' APP V717I } \\
\text { 'Iranian' APP T714A } \\
\text { APP duplication }\end{array}$ & Amyloid angiopathy \& Lewy body pathology \\
\hline Spastic paraparesis & $\begin{array}{l}\text { PSEN1 DelIM } \\
\text { PSEN1 } \triangle \text { exon9 } \\
\text { PSEN1 Y154N } \\
\text { PSEN1 F237I } \\
\text { PSEN1 V261F } \\
\text { PSEN1 P264L } \\
\text { PSEN1 G266S } \\
\text { PSEN1 R278K } \\
\text { PSEN1 R278T } \\
\text { PSEN1 E280G } \\
\text { PSEN1 P284L } \\
\text { PSEN1 P436Q }\end{array}$ & $\begin{array}{l}\text { Cotton wool plaques } \\
\text { Cotton wool plaques } \\
\text { Cotton wool plaques } \\
\text { Cotton wool plaques } \\
\text { Cotton wool plaques } \\
\text { Cotton wool plaques }\end{array}$ \\
\hline Extrapyramidal signs & $\begin{array}{l}\text { PSEN1 S170F } \\
\text { PSEN1 G217D } \\
\text { PSEN1 M223V } \\
\text { PSEN1 DelT440 } \\
\text { PSEN2 A85V }\end{array}$ & $\begin{array}{l}\text { Lewy body pathology } \\
\text { Cotton wool plaques \& amyloid angiopathy } \\
\text { Lewy body pathology } \\
\text { Cotton wool plaques \& Lewy body pathology } \\
\text { Lewy body pathology }\end{array}$ \\
\hline Cerebellar signs & $\begin{array}{l}\text { PSEN1 P117A } \\
\text { PSEN1 S170F }\end{array}$ & Severe Purkinje cell somata and dendritic loss \\
\hline $\begin{array}{l}\text { Focal neurological } \\
\text { symptoms \& signs }\end{array}$ & $\begin{array}{l}\text { 'Dutch' APP E693Q } \\
\text { 'Flemish' APP A692G }\end{array}$ & $\begin{array}{l}\text { Amyloid angiopathy with recurrent cerebral } \\
\text { haemorrhages }\end{array}$ \\
\hline
\end{tabular}




\section{Influence of mutation location on pathology and phenotype}

\section{Presenilin 1 mutations}

Two distinct histopathological profiles have been identified in FAD caused by PSEN1 mutations and the type of pathology appears to be driven by the position of the mutation within the gene. ${ }^{(11)}$ Mutations occurring before codon 200 are more frequently associated with a type1 pattern of pathology whilst mutations beyond codon 200 tend to have type 2 pathology, which includes more severe amyloid angiopathy. Type 1 pathology more closely resembles the pathology seen in sporadic $\mathrm{AD}$, with many diffuse and cored plaques and few white matter plaques. Amyloid angiopathy is only mild to moderate, and is usually confined to leptomeningeal vessels. In type 2 pathology, the number and distribution of diffuse and cored plaques is similar but these tend to be much larger and concentrated around amyloidotic arteries. Amyloid angiopathy is nearly always severe, involving both leptomeningeal and intraparenchymal arteries. A $\beta 40$ deposition tends to be increased in those with type 2 pathology, and this effect is independent of APOE genotype. The distinction between the two types of pathology is not absolute; some mutations occurring before codon 200 have been associated with widespread amyloid angiopathy, such as E184D ${ }^{(62)}$ and DelI83/M84. ${ }^{(63)}$ Equally, the N405S mutation, occurring after codon 200, appears to produce limited amyloid angiopathy. ${ }^{(64)}$ However, there do appear to be clinical implications of the different histopathological signatures. Individuals with type 1 pathology have a younger age at onset (by on average 5 years) and shorter disease duration (by around 2.5 years) than those with type 2 pathology.

In order to explain the dichotomous manner by which the mutation site influences the development of amyloid angiopathy, it has been suggested that PSEN1 mutations may actually act through two separate pathways. ${ }^{(11)}$ First, they increase the production and deposition of $A \beta 42$ and secondly, by affecting Notch signalling, they induce breakdown of the vascular epithelium. The subsequent leakage of $A \beta$ into brain tissue may seed onto existing deposits of $\mathrm{A} \beta$, producing the large plaques seen around vessels in type 2 pathology, and promoting the development of amyloid angiopathy. Interestingly, the residues of PSEN1 that are thought to be functionally most important, namely aspartyl residues at the active site and PSEN1 cleavage site, are 
located in the region of the gene where mutations are associated with severe amyloid angiopathy. Also important is the observation that these type 2 cases have less aggressive phenotypes, with later ages at onset and longer disease durations.

\section{APP mutations}

The majority of APP mutations reported to date lie in the vicinity of the $\beta$ and $\gamma$ secretase cleavage sites. APP residue 717, located just distal to the C-terminus of the $\mathrm{A} \beta$ domain by the $\gamma$ secretase site, was recognised early on to represent a mutation hotspot. The 'London' APP V717I mutation was the first described, comprising valine substituted by isoleucine, but valine substituted by glycine, phenylananine or leucine at the 717 codon also results in a similar phenotype. Families with mutations at this site were found to share common features of early impairment of episodic memory, dyscalculia, lack of insight and prominent myoclonus and seizures. ${ }^{(65)} \mathrm{A}$ double point mutation at APP 670/671 ( $\mathrm{G}$ to $\mathrm{T}$, A to $\mathrm{C}$ ), resulting in a lysine methionine to asparagine leucine double substitution, was later identified in two Swedish families. ${ }^{(66)}$ This double mutation frames the other end of the $\mathrm{A} \beta$ domain, lying at the $\mathrm{N}$-terminus by the $\beta$ secretase site.

Some mutations have also been found within the $A \beta$ coding sequence, and these appear to have specific pathological and clinical features, particularly in relation to amyloid angiopathy (Table 2). The 'Dutch mutation' in APP; a missense mutation resulting in a glutamate to glutamine substitution at position 693 (APP E693Q) gives rise to a highly distinct phenotype. ${ }^{(67)}$ These individuals have severe amyloid angiopathy leading to recurrent cerebral haemorrhage. The main clinical features are focal neurological symptoms and signs relating to these cerebrovascular events, although most patients do also develop dementia. Pathologically, diffuse parenchymal deposits of $A \beta$ are observed, but the hallmark neuritic plaques and neurofibrillary tangles of AD are absent. A neighbouring mutation at residue 692 (an alanine to glycine substitution) has been reported in a separate family and labelled the Flemish mutation. ${ }^{(68)}$ The affected individuals suffered either haemorrhages or a progressive dementia or both. A biopsy of a family member with haemorrhages revealed amyloid angiopathy with sparse neuritic plaques but no neurofibrillary tangles. 
Presymptomatic carriers of the Flemish mutation have also been investigated and found to have significantly increased periventricular and subcortical white matter lesions on cranial MRI. ${ }^{(69)}$

Another APP mutation within the A $\beta$ sequence, known as the Iowa mutation (APP D694N) has also been found to be associated with severe amyloid angiopathy on pathological investigation. Widespread neurofibrillary tangles and amyloid plaques with particularly abundant $A \beta 40$ deposition are seen with this mutation. Clinically, however, these affected individuals lacked symptoms of recurrent cerebrovascular events and presented with progressive cognitive impairment. ${ }^{(70)}$ A similar picture was found with the recently identified 'Arctic' mutation APP E693G, which has a purely cognitive phenotype, typical of $\mathrm{AD}$, despite the presence of marked amyloid angiopathy. ${ }^{(71)}$ The pathological findings in the Arctic mutation also include neurofibrillary tangles and abundant amyloid plaques with characteristic ring-like structures. Why the presence of amyloid angiopathy should have clinical manifestations with some of these mutations but not with others is intriguing. This is particularly true for the Arctic and Dutch mutations, which occur at exactly the same position (amino acid 693) but give rise to totally different phenotypes. It has been proposed that the explanation may lie in polarity of the substituted amino acid. The Dutch mutation involves replacement of a glutamic acid with glutamine, which is polar and hydrophilic. Dutch $A \beta$ may therefore have a greater affinity for, and propensity to disrupt, the vessel wall than Arctic $A \beta$, which is non-polar and hydrophobic. $^{(71)}$ 
Table 2: Clinical and pathological features of APP mutations lying within the $A \beta$ coding domain

\begin{tabular}{|c|c|c|c|c|}
\hline $\begin{array}{l}\text { APP } \\
\text { Mutation }\end{array}$ & $\begin{array}{l}\text { Cerebral } \\
\text { amyloid } \\
\text { angiopathy }\end{array}$ & $\begin{array}{l}\text { Amyloid } \\
\text { plaques }\end{array}$ & $\begin{array}{l}\text { Neurofibrillary } \\
\text { tangles }\end{array}$ & Clinical Phenotype \\
\hline $\begin{array}{l}\text { Flemish } \\
\text { A692G }\end{array}$ & Present & Present & Absent & $\begin{array}{l}\text { Recurrent cerebral } \\
\text { haemorrhage or dementia or } \\
\text { both }\end{array}$ \\
\hline $\begin{array}{l}\text { Dutch } \\
\text { E693Q }\end{array}$ & Present & Absent & Absent & $\begin{array}{l}\text { Predominantly recurrent } \\
\text { cerebral haemorrhage. } \\
\text { Usually also followed by } \\
\text { dementia }\end{array}$ \\
\hline $\begin{array}{l}\text { Arctic } \\
\text { E693G }\end{array}$ & Present & $\begin{array}{l}\text { Present, with } \\
\text { characteristic } \\
\text { ring-like } \\
\text { structure }\end{array}$ & Present & Dementia only \\
\hline $\begin{array}{l}\text { Iowa } \\
\text { D694N }\end{array}$ & Present & $\begin{array}{l}\text { Present, with } \\
\text { extensive } \\
\text { A } \beta 40 \\
\text { deposition }\end{array}$ & Present & Dementia only \\
\hline
\end{tabular}

\section{APP duplications}

The well established link between Down's syndrome and AD provided early evidence that copy number variation may play a role in $\mathrm{AD}$ pathogenesis and it is now known that duplications of the APP gene can give rise to FAD. ${ }^{(4)}$ The phenotype associated with APP duplication can be diverse, even within a single family. ${ }^{(72)}$ However, characteristic features include a high frequency of seizures, up to 57\% in one series, and prominent amyloid angiopathy. ${ }^{(4)}$ The size of the duplication does not appear to influence the clinical presentation. The cognitive phenotype resembles that of typical $\mathrm{AD}$, with early progressive impairment of episodic memory. In some families, the clinical presentation is solely cognitive, with no distinguishing neurological 
features. ${ }^{(73)}$ Knowledge of the prevalence of APP duplications and spectrum of associated clinical features is likely to expand as increasing numbers of centres search for copy number variation in their cohorts of patients.

Interestingly, a recent post-mortem study of an individual with an APP duplication revealed diffuse neocortical Lewy body pathology (LBP) with additional amyloid angiopathy and AD pathology. ${ }^{(72)}$ The clinical phenotype of this patient, comprising cognitive decline associated with visual hallucinations and extrapyramidal signs, was also compatible with a diagnosis of dementia with Lewy bodies. As the authors suggest, this case adds weight to the hypothesis that accumulation of $\mathrm{A} \beta$ can induce LBP. However, further work must be done in larger cohorts of patients to elucidate more about the clinical manifestations of coexistent Lewy body pathology in FAD, and to investigate the relationship between $\beta$-amyloid and alpha-synuclein in neurodegenerative disease in general.

\section{$\underline{\text { Genetic factors in sporadic AD }}$}

In FAD, genetic factors clearly modify the clinical phenotype in striking ways. The manifestations of sporadic AD are also likely to be influenced by genetic factors, though generally in a far subtler manner. The involvement of the APOE gene in sporadic $\mathrm{AD}$ has long been known, with possession of the $\mathrm{E} 4$ allele conferring both an increased risk of developing the disease and possibly driving the pathology towards limbic areas. ${ }^{(74)}$ Genome-wide association studies have recently revealed three new 'risk genes' for sporadic AD: CLU, PICALM and CR1. ${ }^{(75)}$, ${ }^{(76)}$ An important direction for future work will be to investigate whether these variants have phenotypic associations, particularly in sporadic AD but also in familial disease.

Genes known to cause FAD may also play a role in sporadic disease. There are certain PSEN1 polymorphisms, for example E318G, whose pathogenic nature remains uncertain. It has been suggested that some PSEN1 polymorphisms may modify the phenotype of sporadic AD by altering the rate of cognitive decline. ${ }^{(77)}$ 
There is recent evidence demonstrating that FAD-causing gene mutations may sometimes be even more directly implicated in cases of apparently sporadic AD; through somatic and germline mosaicism. ${ }^{(78)}$ An individual who underwent negative genetic testing on a peripheral blood sample was subsequently found to have the PSEN1 P436Q mutation in DNA extracted from cerebral cortex. Although this proband had no family history of dementia herself, one of her daughters subsequently developed symptoms and was found to have inherited the mutant haplotype from her mother. Interestingly, the two affected individuals had a difference in age at onset of around 15 years and strikingly different clinical phenotypes, although both featured prominent neurological signs. As the authors suggest, these findings have several important implications. Differing levels of mosaicism may account for some of the phenotypic variation seen in FAD. Furthermore, there may be many patients with AD who have pathogenic somatic mutations expressed in brain tissue that are not found on conventional sequencing of DNA extracted from blood lymphocytes.

\section{Conclusion}

In the past two decades, significant advances have been made in our understanding of FAD at a molecular, genetic, pathological and clinical level. The number of known mutations has risen exponentially and, with them, an expanding spectrum of clinical phenotypes has been identified. Observations of how certain symptoms and signs tend to cluster with particular mutations has led to an appreciation of how the location of a mutation within a gene can dictate the type of pathology that develops, particularly in relation to amyloid angiopathy. Familial and sporadic AD have been shown to share many similarities. They commonly present with identical symptoms of impaired episodic memory but can both, more rarely, have initial deficits in other cognitive domains. The factors dictating the topography of pathology in both familial and sporadic disease remain to be elucidated. However, possible mechanisms have been proposed. As has always been the case, the insights gained from studying FAD continue to inform our understanding of $\mathrm{AD}$ in general. With the prospect of diseasemodifying therapy focussing interest on detecting the earliest stages of disease, longitudinal study of the presymptomatic phase in individuals destined to develop FAD will be more important than ever. 


\section{Future perspective}

There is a growing appreciation of how knowledge gleaned from the study of familial Alzheimer's disease has the potential to increase our understanding of Alzheimer's disease as a whole. In recognition of this, the first international collaborative study of individuals carrying FAD mutations has recently been launched; the dominantly inherited Alzheimer network (DIAN). This multicentre study will collect longitudinal data to explore the clinical, imaging, neuropsychological, blood and cerebrospinal fluid changes that occur in the long period leading up to symptom onset in FAD. By establishing the largest cohort of FAD patients studied to date, it will allow some of the unresolved questions surrounding the relationship between genotype and phenotype in FAD to be addressed. Aided by initiatives like DIAN, research into FAD is likely to progress at an accelerating pace over the coming decade. The advent of disease-modifying therapy provides great momentum for driving forward this progress, as it creates an unprecedented need to understand the pathogenesis of $\mathrm{AD}$ in more detail. Issues such as the relationship between AD pathology and amyloid angiopathy, for example, have been brought to the forefront by recent immunotherapy trials. It has been found that, in patients treated with A $\beta 42$ immunotherapy, removal of amyloid plaques may be accompanied by accumulation of $A \beta 42$ in perivascular drainage pathways, with an associated transient increase in amyloid angiopathy. ${ }^{(79)} \mathrm{It}$ appears that this plaque-derived $A \beta 42$ can be cleared, given sufficient time. ${ }^{(80)}$ However, factors including APOE genotype are likely to influence how effectively this is achieved. Understanding more about the mechanisms underlying the variable occurrence and clinical manifestation of amyloid angiopathy in FAD may thus have far-reaching implications. It is clear that in both familial and sporadic $\mathrm{AD}$, a range of atypical clinical phenotypes can occur and by understanding more about how genetic factors modify the pathological and clinical picture, we may find that the phenotype contains clues suggesting the most appropriate treatment for an individual. 


\section{$\underline{\text { Executive summary }}$}

\section{Autosomal dominantly inherited genetic changes can cause early onset AD}

- Mutations in the amyloid precursor protein (APP), presenilin 1 (PSEN1) and presenilin 2 (PSEN2) genes, together with APP duplications, may give rise to familial Alzheimer's disease (FAD).

- Fewer mutations have been identified in PSEN2 or APP than PSEN1, which accounts for the majority of cases of FAD.

- FAD accounts for only up to $0.5 \%$ of all Alzheimer's disease.

- Study of FAD gene mutations has informed much of our current knowledge of AD pathogenesis and has facilitated the development of putative treatments.

\section{Role of genetic mutations in AD pathogenesis}

- All of the genetic changes known to cause FAD ultimately result in enhanced production and/or deposition of $\beta$-amyloid.

- Consequently, individuals with FAD generally have much earlier ages at onset of disease than those with sporadic AD.

- The youngest ages at onset are seen with mutations in PSEN1, which may also be associated with shorter disease durations. Older ages at onset occur with APP mutations and the oldest with PSEN2.

- Other genetic and epigenetic factors may contribute to the range in age at onset and variable phenotype that is witnessed both between and within families with the same mutation.

- The APOE4 allele is associated with younger age at onset in sporadic AD and FAD secondary to APP mutations, though the relationship in PSEN1associated FAD is less clear.

\section{Cognitive phenotype of familial and sporadic AD}

- FAD typically presents with insidious impairment of episodic memory, which may be comparable to the amnestic MCI prodrome of sporadic AD.

- Presymptomatic changes of impaired verbal memory and performance IQ, and accelerating brain atrophy on MRI, have been detected in FAD. 
- Atypical presentations, involving other cognitive domains prior to memory, occur in familial and sporadic AD. Language and behavioural variants are seen in both but the visual variant of posterior cortical atrophy has only been reported to occur in sporadic disease.

\section{Neurological symptoms and signs in FAD}

- Neurological symptoms and signs occur more frequently in familial than sporadic AD, particularly in very early onset cases, before the age of 40y.

- Myoclonus and seizures can occur early in the disease course and may precede cognitive symptoms.

- PSEN1 mutations may be associated with spastic paraparesis, extrapyramidal signs or cerebellar ataxia and these may rarely be the presenting features.

- Spastic paraparesis is often associated with PSEN1 exon 9 deletions. Characteristic histopathological features called cotton wool plaques are frequently seen in these cases. These have also been found in PSEN1associated FAD presenting with Parkinsonism.

- Severe cerebellar A $\beta$ deposition has been observed in some PSEN1 and APP717 mutations.

\section{Influence of mutation location on pathology and phenotype}

- PSEN1 mutations before codon 200 have similar histopathology to sporadic $\mathrm{AD}$ whilst those beyond it produce more severe amyloid angiopathy, though a later and longer disease course.

- The 'Dutch' and 'Flemish' APP mutations located within the A $\beta$ coding sequence cause severe amyloid angiopathy with recurrent haemorrhage and focal neurological symptoms and signs. Neighbouring 'Iowa' and 'Arctic' APP mutations also show amyloid angiopathy, although the phenotype is purely cognitive.

- Duplications of the APP gene can cause FAD, often with prominent amyloid angiopathy and a high seizure frequency.

- Concomitant Lewy body pathology occurs in up to $60 \%$ of familial and sporadic AD and may also be seen with APP duplications, suggesting it may 
be induced by $\mathrm{A} \beta$ deposition. There may be clinical correlations of visual hallucinations and Parkinsonism.

\section{Genetic factors in sporadic AD}

- The APOE4 genotype is a well established risk factor for sporadic AD but three additional risk genes, CLU, PICALM and CR1 have also recently been identified.

- Genes known to cause FAD may also play a role in sporadic disease; it has been suggested that PSEN1 polymorphisms may affect the rate of cognitive decline.

- Cases of apparently sporadic AD may sometimes be caused by PSEN1 mutations through somatic and germline mosaicism. Differing levels of mosaicism may also contribute to phenotypic heterogeneity.

\section{Financial disclosure/Acknowledgements}

This work was undertaken at UCL/UCLH which received a proportion of funding from the Department of Health's NIHR Biomedical Research Centres funding scheme. The Dementia Research Centre is an Alzheimer's Research Trust Coordination Centre. NR is supported by a Clinical Research Training Fellowship from the Medical Research Council (UK). MR is a site PI for the NIA funded DIAN study, which receives additional support from DeNDRON, the NIHR clinical research network for dementia and neurodegenerative diseases. The authors have no other relevant affiliations or financial disclosures. 


\section{Reference List}

1. Goate A, Chartier-Harlin MC, Mullan M et al: Segregation of a missense mutation in the amyloid precursor protein gene with familial Alzheimer's disease. Nature 349(6311), 704-706 (1991).

2. Sherrington R, Rogaev EI, Liang Y et al: Cloning of a gene bearing missense mutations in early-onset familial Alzheimer's disease. Nature 375(6534), 754-760 (1995).

3. Levy-Lahad E, Wasco W, Poorkaj P et al: Candidate gene for the chromosome 1 familial Alzheimer's disease locus. Science 269(5226), 973-977 (1995).

4. Rovelet-Lecrux A, Hannequin D, Raux G et al: APP locus duplication causes autosomal dominant early-onset Alzheimer disease with cerebral amyloid angiopathy. Nat.Genet. 38(1), 24-26 (2006).

5. Larner AJ , Doran M: Clinical phenotypic heterogeneity of Alzheimer's disease associated with mutations of the presenilin-1 gene. J Neurol 253(2), 139-158 (2006).

6. Larner AJ , Doran M: Genotype-phenotype relationships of presenilin-1 mutations in Alzheimer's disease: an update. J Alzheimers.Dis 17(2), 259-265 (2009).

7. Selkoe DJ: Alzheimer's disease: genotypes, phenotypes, and treatments. Science 275(5300), 630-631 (1997).

8. Nilsberth C, Westlind-Danielsson A, Eckman CB et al: The 'Arctic' APP mutation (E693G) causes Alzheimer's disease by enhanced Abeta protofibril formation. Nat.Neurosci 4(9), 887-893 (2001).

9. Holmes C: Genotype and phenotype in Alzheimer's disease. Br.J Psychiatry 180, 131-134 (2002).

10. Godbolt AK, Cipolotti L, Watt H, Fox NC, Janssen JC, Rossor MN: The natural history of Alzheimer disease: a longitudinal presymptomatic and symptomatic study of a familial cohort. Arch Neurol 61(11), 17431748 (2004).

11. Mann DM, Pickering-Brown SM, Takeuchi A, Iwatsubo T: Amyloid angiopathy and variability in amyloid beta deposition is determined by mutation position in presenilin-1-linked Alzheimer's disease. Am J Pathol. 158(6), 2165-2175 (2001).

12. Van Broeckhoven $\mathrm{C}$, Backhovens $\mathrm{H}$, Cruts $\mathrm{M}$ et al: APOE genotype does not modulate age of onset in families with chromosome 14 encoded Alzheimer's disease. Neurosci Lett. 169(1-2), 179-180 (1994). 
13. Pastor P, Roe CM, Villegas A et al: Apolipoprotein Eepsilon4 modifies Alzheimer's disease onset in an E280A PS1 kindred. Ann Neurol 54(2), 163-169 (2003).

14. Fox NC, Warrington EK, Seiffer AL, Agnew SK, Rossor MN:

Presymptomatic cognitive deficits in individuals at risk of familial Alzheimer's disease. A longitudinal prospective study. Brain 121 ( Pt 9), 1631-1639 (1998).

15. Warrington EK, Agnew SK, Kennedy AM, Rossor MN: Neuropsychological profiles of familial Alzheimer's disease associated with mutations in the presenilin 1 and amyloid precursor protein genes. J Neurol 248(1), 45-50 (2001).

16. Scahill RI, Schott JM, Stevens JM, Rossor MN, Fox NC: Mapping the evolution of regional atrophy in Alzheimer's disease: unbiased analysis of fluid-registered serial MRI. Proc Natl.Acad.Sci.U.S.A 99(7), 47034707 (2002).

17. Chan D, Janssen JC, Whitwell JL et al: Change in rates of cerebral atrophy over time in early-onset Alzheimer's disease: longitudinal MRI study. Lancet 362(9390), 1121-1122 (2003).

18. Petersen RC, Smith GE, Waring SC, Ivnik RJ, Tangalos EG, Kokmen E: Mild cognitive impairment: clinical characterization and outcome. Arch Neurol 56(3), 303-308 (1999).

19. Godbolt AK, Beck JA, Collinge $\mathrm{J}$ et al: A presenilin 1 R278I mutation presenting with language impairment. Neurology 63(9), 1702-1704 (2004).

20. Gorno-Tempini ML, Brambati SM, Ginex V et al: The logopenic/phonological variant of primary progressive aphasia. Neurology 71(16), 1227-1234 (2008).

21. Marcon G, Di Fede G, Giaccone G et al: A novel Italian presenilin 2 gene mutation with prevalent behavioral phenotype. J Alzheimers.Dis 16(3), 509-511 (2009).

22. Queralt R, Ezquerra M, Lleo A et al: A novel mutation (V89L) in the presenilin 1 gene in a family with early onset Alzheimer's disease and marked behavioural disturbances. J Neurol Neurosurg Psychiatry 72(2), 266-269 (2002).

23. Zekanowski C, Golan MP, Krzysko KA et al: Two novel presenilin 1 gene mutations connected with frontotemporal dementia-like clinical phenotype: genetic and bioinformatic assessment. Exp.Neurol 200(1), 82-88 (2006).

24. Rippon GA, Crook R, Baker M et al: Presenilin 1 mutation in an african american family presenting with atypical Alzheimer dementia. Arch Neurol 60(6), 884-888 (2003). 
25. Raux G, Gantier R, Thomas-Anterion C et al: Dementia with prominent frontotemporal features associated with L113P presenilin 1 mutation. Neurology 55(10), 1577-1578 (2000).

26. Amtul Z, Lewis PA, Piper S et al: A presenilin 1 mutation associated with familial frontotemporal dementia inhibits gamma-secretase cleavage of APP and notch. Neurobiol Dis 9(2), 269-273 (2002).

27. Tang-Wai D, Lewis $\mathrm{P}$, Boeve B et al: Familial frontotemporal dementia associated with a novel presenilin-1 mutation. Dement Geriatr Cogn Disord 14(1), 13-21 (2002).

28. Pickering-Brown SM, Baker M, Gass $\mathrm{J}$ et al: Mutations in progranulin explain atypical phenotypes with variants in MAPT. Brain 129(Pt 11), 31243126 (2006).

29. Mendez MF, Ghajarania M, Perryman KM: Posterior cortical atrophy: clinical characteristics and differences compared to Alzheimer's disease. Dement Geriatr Cogn Disord 14(1), 33-40 (2002).

30. Snider BJ, Norton J, Coats MA et al: Novel presenilin 1 mutation (S170F) causing Alzheimer disease with Lewy bodies in the third decade of life. Arch Neurol 62(12), 1821-1830 (2005).

31. Kennedy AM, Newman S, McCaddon A et al: Familial Alzheimer's disease. A pedigree with a mis-sense mutation in the amyloid precursor protein gene (amyloid precursor protein 717 valine-->glycine). Brain $116(\mathrm{Pt}$ 2), 309-324 (1993).

32. Campion D, Brice A, Dumanchin C et al: A novel presenilin 1 mutation resulting in familial Alzheimer's disease with an onset age of 29 years. Neuroreport 7(10), 1582-1584 (1996).

33. Karlstrom H, Brooks WS, Kwok JB et al: Variable phenotype of Alzheimer's disease with spastic paraparesis. J Neurochem. 104(3), 573-583 (2008).

34. Assini A, Terreni L, Borghi R et al: Pure spastic paraparesis associated with a novel presenilin 1 R278K mutation. Neurology 60(1), 150- (2003).

35. Hiltunen M, Helisalmi S, Mannermaa A et al: Identification of a novel 4.6-kb genomic deletion in presenilin-1 gene which results in exclusion of exon 9 in a Finnish early onset Alzheimer's disease family: an Alu core sequence-stimulated recombination? Eur J Hum.Genet. 8(4), 259-266 (2000).

36. Verkkoniemi A, Somer M, Rinne JO et al: Variant Alzheimer's disease with spastic paraparesis: clinical characterization. Neurology 54(5), $1103-$ 1109 (2000).

37. O'Riordan S, McMonagle P, Janssen JC et al: Presenilin-1 mutation (E280G), spastic paraparesis, and cranial MRI white-matter abnormalities. Neurology 59(7), 1108-1110 (2002). 
38. Houlden H, Baker M, McGowan E et al: Variant Alzheimer's disease with spastic paraparesis and cotton wool plaques is caused by PS-1 mutations that lead to exceptionally high amyloid-beta concentrations. Ann Neurol 48(5), 806-808 (2000).

39. Tabira T, Chui DH, Nakayama H, Kuroda S, Shibuya M: Alzheimer's disease with spastic paresis and cotton wool type plaques. J Neurosci Res. 70(3), 367-372 (2002).

40. Yokota $\mathrm{O}$, Terada S, Ishizu $\mathrm{H}$ et al: Variability and heterogeneity in Alzheimer's disease with cotton wool plaques: a clinicopathological study of four autopsy cases. Acta Neuropathol 106(4), 348-356 (2003).

41. Dintchov TL, Mehrabian S, Van den BM et al: Novel PSEN1 mutation in a bulgarian patient with very early-onset Alzheimer's disease, spastic paraparesis, and extrapyramidal signs. Am J Alzheimers.Dis Other Demen. 24(5), 404-407 (2009).

42. Piscopo P, Marcon G, Piras MR et al: A novel PSEN2 mutation associated with a peculiar phenotype. Neurology 70(17), 1549-1554 (2008).

43. Takao M, Ghetti B, Hayakawa I et al: A novel mutation (G217D) in the Presenilin 1 gene ( PSEN1) in a Japanese family: presenile dementia and parkinsonism are associated with cotton wool plaques in the cortex and striatum. Acta Neuropathol 104(2), 155-170 (2002).

44. Ishikawa A, Piao YS, Miyashita A et al: A mutant PSEN1 causes dementia with Lewy bodies and variant Alzheimer's disease. Ann Neurol 57(3), 429-434 (2005).

45. Houlden H, Crook R, Dolan RJ, McLaughlin J, Revesz T, Hardy J: A novel presenilin mutation (M233V) causing very early onset Alzheimer's disease with Lewy bodies. Neurosci Lett. 313(1-2), 93-95 (2001).

46. Lippa CF, Fujiwara H, Mann DM et al: Lewy bodies contain altered alphasynuclein in brains of many familial Alzheimer's disease patients with mutations in presenilin and amyloid precursor protein genes. Am J Pathol. 153(5), 1365-1370 (1998).

47. Hamilton RL: Lewy bodies in Alzheimer's disease: a neuropathological review of 145 cases using alpha-synuclein immunohistochemistry. Brain Pathol. 10(3), 378-384 (2000).

48. Leverenz JB, Fishel MA, Peskind ER et al: Lewy body pathology in familial Alzheimer disease: evidence for disease- and mutation-specific pathologic phenotype. Arch Neurol 63(3), 370-376 (2006).

49. Cole G, Williams P, Alldryck D, Singharo S: Amyloid plaques in the cerebellum in Alzheimer's disease. Clin Neuropathol 8(4), 188-191 (1989). 
50. Larner AJ: The cerebellum in Alzheimer's disease. Dement Geriatr Cogn Disord 8(4), 203-209 (1997).

51. Lemere CA, Lopera F, Kosik KS et al: The E280A presenilin 1 Alzheimer mutation produces increased A beta 42 deposition and severe cerebellar pathology. Nat.Med 2(10), 1146-1150 (1996).

52. Martin JJ, Gheuens J, Bruyland M et al: Early-onset Alzheimer's disease in 2 large Belgian families. Neurology 41(1), 62-68 (1991).

53. Haltia M, Viitanen M, Sulkava R et al: Chromosome 14-encoded Alzheimer's disease: genetic and clinicopathological description. Ann Neurol 36(3), 362-367 (1994).

54. Lampe TH, Bird TD, Nochlin D et al: Phenotype of chromosome 14-linked familial Alzheimer's disease in a large kindred. Ann Neurol 36(3), 368378 (1994).

55. Mann DM, Iwatsubo T, Ihara Y et al: Predominant deposition of amyloid-beta 42(43) in plaques in cases of Alzheimer's disease and hereditary cerebral hemorrhage associated with mutations in the amyloid precursor protein gene. Am J Pathol. 148(4), 1257-1266 (1996).

56. Mann DM, Jones D, Prinja D, Purkiss MS: The prevalence of amyloid (A4) protein deposits within the cerebral and cerebellar cortex in Down's syndrome and Alzheimer's disease. Acta Neuropathol 80(3), 318-327 (1990).

57. Mann DM, Iwatsubo T: Diffuse plaques in the cerebellum and corpus striatum in Down's syndrome contain amyloid beta protein (A beta) only in the form of A beta 42(43). Neurodegeneration. 5(2), 115-120 (1996).

58. Fukutani Y, Cairns NJ, Rossor MN, Lantos PL: Cerebellar pathology in sporadic and familial Alzheimer's disease including APP 717 (Val->Ile) mutation cases: a morphometric investigation. J Neurol Sci. 149(2), 177-184 (1997).

59. Piccini A, Zanusso G, Borghi R et al: Association of a presenilin 1 S170F mutation with a novel Alzheimer disease molecular phenotype. Arch Neurol 64(5), 738-745 (2007).

60. Anheim M, Hannequin D, Boulay C, Martin C, Campion D, Tranchant C: Ataxic variant of Alzheimer's disease caused by Pro117Ala PSEN1 mutation. J Neurol Neurosurg Psychiatry 78(12), 1414-1415 (2007).

61. Dermaut B, Kumar-Singh S, De Jonghe C et al: Cerebral amyloid angiopathy is a pathogenic lesion in Alzheimer's disease due to a novel presenilin 1 mutation. Brain 124(Pt 12), 2383-2392 (2001).

62. Yasuda M, Maeda K, Ikejiri Y, Kawamata T, Kuroda S, Tanaka C: A novel missense mutation in the presenilin-1 gene in a familial Alzheimer's 
disease pedigree with abundant amyloid angiopathy. Neurosci Lett. 232(1), 29-32 (1997).

63. Steiner H, Revesz T, Neumann M et al: A pathogenic presenilin-1 deletion causes abberrant Abeta 42 production in the absence of congophilic amyloid plaques. J Biol.Chem. 276(10), 7233-7239 (2001).

64. Yasuda M, Maeda S, Kawamata T et al: Novel presenilin-1 mutation with widespread cortical amyloid deposition but limited cerebral amyloid angiopathy. J Neurol Neurosurg Psychiatry 68(2), 220-223 (2000).

65. Rossor MN, Newman S, Frackowiak RS, Lantos P, Kennedy AM: Alzheimer's disease families with amyloid precursor protein mutations. Ann $N$ Y.Acad.Sci. 695, 198-202 (1993).

66. Mullan M, Crawford F, Axelman K et al: A pathogenic mutation for probable Alzheimer's disease in the APP gene at the N-terminus of betaamyloid. Nat.Genet. 1(5), 345-347 (1992).

67. Van Broeckhoven C, Haan J, Bakker E et al: Amyloid beta protein precursor gene and hereditary cerebral hemorrhage with amyloidosis (Dutch). Science 248(4959), 1120-1122 (1990).

68. Hendriks L, van Duijn CM, Cras P et al: Presenile dementia and cerebral haemorrhage linked to a mutation at codon 692 of the beta-amyloid precursor protein gene. Nat.Genet. 1(3), 218-221 (1992).

69. Roks G, Van Harskamp F, De K, I et al: Presentation of amyloidosis in carriers of the codon 692 mutation in the amyloid precursor protein gene (APP692). Brain 123 ( Pt 10), 2130-2140 (2000).

70. Grabowski TJ, Cho HS, Vonsattel JP, Rebeck GW, Greenberg SM: Novel amyloid precursor protein mutation in an Iowa family with dementia and severe cerebral amyloid angiopathy. Ann Neurol 49(6), 697-705 (2001).

71. Basun H, Bogdanovic N, Ingelsson $\mathrm{M}$ et al: Clinical and neuropathological features of the arctic APP gene mutation causing early-onset Alzheimer disease. Arch Neurol 65(4), 499-505 (2008).

72. Guyant-Marechal I, Berger E, Laquerriere A et al: Intrafamilial diversity of phenotype associated with app duplication. Neurology 71(23), 19251926 (2008).

73. Kasuga K, Shimohata T, Nishimura A et al: Identification of independent APP locus duplication in Japanese patients with early-onset Alzheimer disease. J Neurol Neurosurg Psychiatry 80(9), 1050-1052 (2009).

74. Schott JM, Ridha BH, Crutch SJ et al: Apolipoprotein e genotype modifies the phenotype of Alzheimer disease. Arch Neurol 63(1), 155-156 (2006). 
75. Harold D, Abraham R, Hollingworth $\mathrm{P}$ et al: Genome-wide association study identifies variants at CLU and PICALM associated with Alzheimer's disease. Nat.Genet. 41(10), 1088-1093 (2009).

76. Lambert JC, Heath S, Even $\mathrm{G}$ et al: Genome-wide association study identifies variants at CLU and CR1 associated with Alzheimer's disease. Nat.Genet. 41(10), 1094-1099 (2009).

77. Belbin O, Beaumont H, Warden D, Smith AD, Kalsheker N, Morgan K: PSEN1 polymorphisms alter the rate of cognitive decline in sporadic Alzheimer's disease patients. Neurobiol Aging 30(12), 1992-1999 (2009).

78. Beck JA, Poulter M, Campbell TA et al: Somatic and germline mosaicism in sporadic early-onset Alzheimer's disease. Hum.Mol.Genet. 13(12), 1219-1224 (2004).

79. Weller RO, Boche D, Nicoll JA: Microvasculature changes and cerebral amyloid angiopathy in Alzheimer's disease and their potential impact on therapy. Acta Neuropathol 118(1), 87-102 (2009).

80. Boche D, Zotova E, Weller RO et al: Consequence of Abeta immunization on the vasculature of human Alzheimer's disease brain. Brain 131(Pt 12), 3299-3310 (2008).

102. http://www.molgen.ua.ac.be/ADMutations

\section{Recommended references}

4. Duplication of the APP locus found to be a cause of autosomal dominantly inherited early onset $\mathrm{AD}$, with prominent amyloid angiopathy.

$5 \& 6$. Comprehensive review of the various different atypical features reported in association with PSEN1 mutations with more recently published update.

11. The histopathological profile in PSEN1 mutations appears to be driven by the position of the mutation in the gene, with more severe amyloid angiopathy observed in mutations beyond codon 200 . 
14. Neuropsychological deficits, manifested in a fall in performance IQ and verbal memory scores, are detectable several years prior to symptoms onset in FAD mutation carriers.

16. Global brain atrophy increases with advancing disease in FAD, and regional hippocampal atrophy is already occurring prior to the onset of symptoms.

33. Detailed review of the association between Alzheimer's disease and spastic paraparesis.

78. This paper introduces somatic and germline mosaicism as novel disease mechanisms in $\mathrm{AD}$, reporting an individual demonstrating a PSEN1 mutation in DNA extracted from cerebral cortex, which was not detectable on peripheral blood sequencing. 